\title{
Health Disparities in Latino Communities
}

\author{
Erualdo R. González \\ California State University, Fullerton
}

\section{Health Disparities, Health Equity, and Social Justice}

The concept of health disparities most often refers to differences in health status and quality of health care across racial, ethnic, gender, and socioeconomic groups. By now, we know that racial and ethnic minorities in the United States, as a whole, have generally poorer health than Whites. Studies of health disparities and efforts to reduce them have traditionally focused on differences in health care access and healthrelated behavior based on race, ethnicity, and culture. For example, interventions may target individuals and groups and attempt to alter health care access and individually controlled lifestyle choices. These efforts include improving the access to and quality of health care and making sure that target populations are integrated into the health care system. Efforts could involve ensuring that the services of doctors and other health professionals are relatively affordable and making sure that health care providers and other practitioners are knowledgeable of and empathetic with cultural backgrounds of their clients and provide culturally appropriate service (e.g., language, educational materials). Thus the chief strategy to reduce health disparities is targeting individuals and groups and the health care system that treats them. In this way, health equity could be seen as the process of reaching individuals in order to reduce disparities in health.

Persistent health disparities in disadvantaged and racial/ethnic communities have propelled additional ways to understand health disparities beyond health equity (Braunstein \& LavizzoMourey, 2011). These include the concepts of social justice and health inequity. Social justice in health focuses on leveling the playing field among populations, such as changing living conditions, policies, and social arrangements that expose some groups to unhealthier environments than others, and providing the same opportunities to make health-promoting choices as those enjoyed by more advantaged groups (Freudenberg, Klitzman, \& Saegert, 2009). Social justice in health is also a matter of addressing health inequity - the avoidable, unnecessary, socially produced, and unfair existence of health status difference among populations (Whitehead \& Dahlgren, 2007). These perspectives take on particular significance because nearly one-fifth of all Americans live in poor neighborhoods, with ethnic minority groups disproportionately accounting for these Americans (Cubin, Pedregon, Egerter, \& Braveman, 2008). Further, ethnic minorities are more likely to live in disadvantaged neighborhoods characterized by inadequate community development (e.g., poor municipal services, lack of open spaces; Fitzpatrick \& LaGory, 2011).

\section{Latino Communities in the United States: A Brief Profile}

According to the 2010 Census, Latinos represent $16.3 \%$ (50.7 million) of the U.S. population, making them the largest minority group. From 2000 to 2010, the Latino population accounted for more than half of the total U.S. population increase of 26.3 million. The demographic growth occurred in all regions and most significantly in non-traditional areas such as the Midwest and South (Motel \& Patten, 2012). Nearly $37 \%$ of all Latinos are immigrants, compared with $13 \%$ of the overall U.S. population (Motel \& Patten, 2012). Populations of Mexican ancestry, followed by Puerto Ricans and Cubans, make up the majority of the Latino population (U.S. Census, 2012). 
Latinos are less likely to have a college degree, likely to earn less than the median income, more likely to live in poverty, and less likely to have health insurance than Whites (Motel \& Patten, 2012). While these statistics are unacceptable, we must also consider additional realities that are common among the Latino unauthorized population.

While almost $75 \%$ of Latinos are U.S. citizens, the unauthorized immigrant population is particularly vulnerable to health disparities (Motel \& Patten, 2012). Immigrants tend to work mainly in the service sector and informal economy, sectors that typically do not provide employer-based insurance coverage and pay low wages. Unauthorized immigrants are virtually excluded from many parts of the health care system.

These statistics would fit with the traditional view of health disparities, particularly the focus on quality, accessibility, and affordability to health care among Latinos. Yet if we are to take health and social justice seriously, we must also consider the contexts in which many Latinos have lived and continue to live. We must continue to bridge community development and health (Corburn, 2007; Gonzalez et al., 2007; Williams \& Marks, 2011). Working-class Latinos in the U.S. have historically lived in places, urban and rural, with inadequate open spaces, physical infrastructure, and other amenities (Diaz, 2005; Irazabal \& Farhat, 2009). The social justice perspective would focus on these conditions as they contribute to health inequity.

\section{Why this Special Issue?}

The Robert Wood Johnson Foundation defines vulnerable populations as those that do not have the same opportunities to make healthy decisions as others, and whose opportunities for good health are exacerbated by insufficient education, inadequate housing, racism, or low income (RWJF, 2012). Latinos may be viewed as one such vulnerable population.

This special issue of the Californian Journal of Health Promotion, "Health Disparities in Latino Communities," contributes to a growing scholarship on health disparities and vulnerable populations. We have assembled a rich collection of manuscripts. The majority of manuscripts utilize a health disparities approach, and several employ a social justice/health equity perspective. Half of the manuscripts focus on obesity, and a similar number span multiple topics, such as mental health and prenatal care. A sample of manuscripts also brings attention to immigration and includes populations in areas that have not traditionally been part of the health disparities literature (e.g., Latinos in the South).

Two program evaluations in this journal bring attention to immigrant experiences. Torres, Smithwick, and Luchok evaluate the PASO program--- a culturally relevant and literacyappropriate parental education intervention designed to help reduce Latino maternal and child health disparities. The evaluation examines prenatal knowledge gain and behavioral change. Participants were 346 Latina women in South Carolina between the ages of 20 and 30 years old, most having lived in the U.S. for less than 10 years. Green et al. provide evaluation results from their pilot of Amigas Latinas Motivando el Alma (ALMA: Latina Friends Motivating the Soul), a culturally relevant training curriculum designed to reduce preclinical depression and anxiety among recent Latina immigrants. The ALMA curriculum is an individual-level intervention to train promotoras in stress-reduction strategies for their own use and to disseminate these strategies within their social networks. Twenty adult self-identified Latinas in North Carolina participated in this pilot study. As a whole, these two evaluations expand the focus on program design and evaluation and highlight some issues that Latina immigrants face.

"Ethnic and Immigration Status Differences on Child Indicators of Health for European Americans and Latinos," by Granillo and colleagues, investigates whether children of immigrants show more positive outcomes than children of U.S. born parents. We see in this work how children of immigrants may be at heightened risk for experiencing poor health (e.g., obesity) compared to the general young population. As noted by the authors, this work 
could inform the design of interventions designed to assist immigrant parents to maintain good health and teach healthy behaviors to their children.

Iland, Weiner, and Murawski's study examines Latina mothers of children with autism. Participants were 96 self-identified Latina mothers of children with autism spectrum disorders (ASD) living in California who were active in parent support organizations. The measures included several surveys. This study brings light to needs and obstacles of Latina mothers dealing with ASD.

Henderson and her colleagues describe their collaboration with community members to tackle obesity in Latino neighborhoods. They used a mixed-methods evaluation tool to document physical activity and nutrition environments at the neighborhood level, and to document community development and public infrastructure needs and aspirations of residents. They used multiple methods to compile data for 15 urban and predominantly Latino neighborhoods in Southern Arizona. This work brings attention to data that neighborhoods could use to plan for policy and environmental changes related to physical activity and nutrition.

"Getting to Know U.S. Latinos: A Step Toward Cultural Competence," by Perez and Luquis, describes an interactive "Jeopardy" style educational program designed to increase knowledge of various health promotion, cultural, culinary, and other practices that are common to the Latino population. The activity is designed for English-speaking audiences and may be used with junior or senior high school or college students. This activity raises attention to the benefits of innovative health education programs for youth.

Rovito and Leone present results from a seminar series designed to promote men's health issues, such as cancer screening. Approximately 70 Latino adult members Philadelphia participated across three focus group discussions. A contribution of this article is the finding that the Latino men reported that they are generally encouraged to be strong and not show the need for health care utilization.

"Sexo y la Ciudad" by Vega, Spieldenner, and Tang explores how urban immigrant Latino gay men negotiate their ethnic, sexual, and social identities. In particular, this study examined these issues in order to tailor an HIV preventionintervention program originally designed for white gay men. The authors used bilingual focus groups with 28 men in New York City. The study is an example of research that strives to inform culturally relevant sexually-oriented health service interventions to reduce or prevent risk behaviors.

Gonzalez, Villanueva, and Grills examine community organizing as an approach to reduce obesity. As part of a national evaluation of the Communities Creating Health Environments (CCHE) of the Robert Wood Johnson Foundation, the authors present preliminary evaluation results from two community-based organizations that are making efforts to increase access to physical activity and access to healthy foods in predominantly Latino areas. This study's contribution is that it provides a community-level evaluation framework and points to the need for public policy research on obesity that examines organizing as an intervention.

Rivera and Burgos' literature review examines best practices of evidence-based interventions designed to reduced BMI among Latina/os of Mexican origin in the U.S. The authors point out that the review is timely, given the obesity epidemic and dearth of literature synthesizing such interventions with this population. Ultimately, the intention of the review is to assist researchers and practitioners, among others, to examine and design obesity related interventions with Latinos.

Jankovitz and colleagues conducted a crosssectional study to determine the prevalence of overweight/obesity (OW/OB) in children ages 3 to 5. A total of 56 Latino and Caucasian participants were part of this study, all from San Luis Obispo County (California) and attending private and public preschools. Measures 
included standardized protocols to measure height and weight. This study raises attention to issues of race and class and recommends schoolbased physical activity and culturally appropriate interventions.

Collectively, this special issue "Health Disparities in Latino Communities" brings attention to topics that require attention in the health disparities and vulnerable populations literature. These topics include race, ethnicity/culture, social subgroups (e.g., unauthorized immigrants), and environments. I hope that this collection of articles assists researchers and practitioners with future directions that could be expanded to help our most vulnerable populations and disadvantaged communities.

\section{References}

Corburn, J. 2007. Reconnecting with our roots: American urban planning and public health in the $21^{\text {st }}$ century. Urban Affairs Review, 42: 688-713.

Cubbin, C., Pedregon, V., Egerter, S., \& Braveman, P. 2008. Where we live matters for our health: Neighborhoods and health. Issue Brief 3: Neighborhoods and Health. Princeton, NJ: Robert Wood Johnson Foundation Commission to Build a Healthier America.

Diaz, D. 2005. Barrio urbanism: Chicanos, planning, and American cities. New York and London: Routledge.

Fitzpatrick, K. \& LaGory, M. 2011. Unhealthy cities: Poverty, race, and place in America. New York and London: Routledge.

Freudenberg, Klitzman, \& Saegert, 2009. Urban health and society: Interdisciplinary approaches to research and practice. San Franciso: John Wiley \& Sons.

González, E. R., Lejano, R. P., Vidales, L., Conner, F. R., Kidokoro, Y., Fazeli, B., \& Cabrales, R. (2007).Participatory action research for environmental health: Encountering Freire in the urban barrio. Journal of Urban Affairs, 29 (1), 77-100.

Irazabal, C., \& Farhat, R. 2008. Latino communities in the United States: Place-making in the pre-World War II, postwar, and contemporary city. Journal of Planning Literature, 22 (207): 207-228.

Motel, S. \& Patten, E. 2012. The 10 Largest Hispanic origin groups: Characteristics, rankings, top counties. http://www.pewhispanic.org/2012/06/27/the-10-largest-hispanic-origin-groupscharacteristics-rankings-top-counties/

Robert Wood Johnson Foundation. 2012. Program areas: Vulnerable populations. http://www.rwjf.org/en/about-rwjf/program-areas/vulnerable-populations.html

U.S. Census. 2012. 2010 census shows nation's Hispanic population grew four times faster than total U.S. population. http://2010.census.gov/news/releases/operations/cb11-cn146.html

Whitehead, M \& Dahlgren, G. 2007. Concepts and principles for tackling social inequities in health: Levelling up Part 1. Copenhagen: World Health Organization.

Williams, D. R. \& Marks, J. 2012. Community development efforts offer a majority opportunity to advance Americans' health. Health Affairs, 30 (11): 2052-2055.

(C) 2012 Californian Journal of Health Promotion. All rights reserved. 